\title{
Advances in Scanning Microwave Impedance Microscopy
}

\section{Ravi Chandra Chintala and Yongliang Yang}

Primenano, Inc., Santa Clara, California, United States

Scanning Microwave Impedance Microscopy (sMIM) is a near-field scanning probe microscopy technique to measure the local dielectric properties of materials. In this technique, an AFM cantilever is used as a microwave source to measure the electrical properties of materials at nanometer scale (Refer Figure 1a for schematic setup). sMIM is sensitive to the local capacitive and conductivity changes in a material making it an excellent method for characterizing wide range of materials such as Insulators, semiconductors, 1D/2D, Ferroelectric materials. In order to reduce background capacitance and, increase the spatial resolution, we employ cantilevers which are shielded on both the sides, exposing only the pyramid of the AFM tip[1]. As the tip scans on the surface, changes in the local permittivity and conductivity can be mapped with nanometer resolution. For semiconductor devices, the measured capacitance can be converted to carrier concentration; to obtain dopant concentration and polarity of the dopant with spatial resolution of < $10 \mathrm{~nm}$. sMIM can operate in various AFM modes such as Tapping, Contact, Non-contact, Peak Force mode[2]. Peak Force and Tapping mode have been used to characterize topography of softer materials. Using sMIM in the above-mentioned modes makes it a valuable tool to study softer materials. Furthermore, since the microwaves can penetrate deeper into the sample surface, we can characterize sub-surface features as well [3,4]. This property is particularly useful when characterizing ambient sensitive materials. A thin passivation layer can be deposited to protect the surface from degradation and sMIM can be used to probe sub-surface features. As sMIM inherently measures the capacitance of the sample, it can be used to study various 2D material systems. It can be used to image Moiré pattern formed between h-BN and graphene with different twist angles. In this talk, we will review the state of the art in sMIM presenting several applications and case studies showing the versatile applicability of sMIM (few examples are shown in Figure 1b).



Figure 1. a) schematic of the sMIM set up b) Example images showcasing the versatile applicability of sMIM

References

1. Lai et al., Applied Nanoscience (2011) 1:13-18 
2. Lai et al., Review of Scientific Instruments 80, 043707 (2009)

3. A. Scrymgeour et al. / Applied Surface Science 423 (2017) 1097-1102

4. Tselev et al., ACS Nano2016, 10, 3, 3562-3570 